Check for updates

Cite this: RSC Adv., 2017, 7, 44043

Received 19th June 2017

Accepted 7th September 2017

DOI: $10.1039 / \mathrm{c} 7 \mathrm{ra06835b}$

rsc.li/rsc-advances

\section{Non-covalent functionalized hexagonal boron nitride nanoplatelets to improve corrosion and wear resistance of epoxy coatings}

\author{
Mingjun Cui, $\uparrow^{\mathrm{ab}}$ Siming Ren, (D) $\dagger^{\mathrm{ab}}$ Songlv Qin, ${ }^{\mathrm{a}}$ Qunji Xue, ${ }^{\mathrm{a}}$ Haichao Zhao*a \\ and Liping Wang*a
}

In the present study, a non-covalent method was employed to modify hexagonal boron nitride ( $h$ - $B N$ ) nanoplatelets through $\pi-\pi$ interaction of amine-capped aniline trimer (AT), which resulted in a stable dispersion of h-BN nanoplatelets in organic solvents. Meanwhile, epoxy coatings containing different content of h-BN nanoplatelets were fabricated, and corresponding corrosion protection performance and wear resistance were investigated. Results showed that the $1.0 \mathrm{wt} \% \mathrm{~h}$-BN/epoxy coating has better corrosion protection performance than other coatings, which was due to the good barrier properties from stably dispersed h-BN nanoplatelets and the passivating effect from AT. Besides, the addition of $\mathrm{h}-\mathrm{BN}$ nanoplatelets also contributed to the improvement in wear resistance of epoxy the coating.

\section{Introduction}

Since successful exfoliation of monolayer graphene from graphite in 2004, there has been a recent explosion of interest in synthesis and applications of two dimensional (2D) materials. Particularly, hexagonal boron nitride (h-BN), one of 2D materials analogous to graphene, shares similar properties with graphene, like high mechanical strength, ${ }^{\mathbf{1}}$ thermal conductivity, ${ }^{1-4}$ oxidation resistance ${ }^{5,6}$ and ultrahigh impermeable properties. $^{7,8}$ However, h-BN possesses unique properties as well. The reduced electro-delocalization in $\mathrm{B}-\mathrm{N}$ bonds leads to an electrically insulating nature, making h-BN a useful filler to improve the thermal conductivity and electrical insulation of polymers. ${ }^{9}$ In addition, the extra barrier property of h-BN makes it suitable as protective materials against metal corrosion and oxidation under aggressive environments. For instance, Liu et $a .^{\mathbf{1 0}}$ had recently reported on the ultrathin h-BN films on $\mathrm{Ni}$ foils by chemical vapor deposition (CVD) method, which was impervious to oxygen diffusion even at $1100{ }^{\circ} \mathrm{C}$ and thus h-BN could serve as oxidation-resistant coatings in oxygen-rich atmosphere. Shen and coworkers ${ }^{7}$ compared the corrosion resistance of h-BN and graphene coating on $\mathrm{Cu}$ through theoretical and experimental method, and concluded that h-BN coating showed much long-term corrosion barrier property

\footnotetext{
${ }^{a}$ Key Laboratory of Marine Materials and Related Technologies, Zhejiang Key Laboratory of Marine Materials and Protective Technologies, Ningbo Institute of Materials Technology and Engineering, Chinese Academy of Sciences, Ningbo 315201, P. R. China. E-mail: zhaohaichao@nimte.ac.cn; wangliping@nimte.ac.cn; Fax: +86-574-86685159; Tel: +86-574-86657094

${ }^{b}$ University of Chinese Academy of Sciences, Beijing 100039, China

$\dagger$ These authors contributed equally to this work.
}

than graphene coating since the high insulated feature of h-BN suppressed the electrochemical reaction. ${ }^{7}$ An alternative strategy for achieving the wide application of h-BN with high performance is introducing h-BN into polymer matrix to form a composite coating that combines the advantages of two components. Esam Husain and coworkers ${ }^{8}$ reported that h-BN/ polymer coating showed an effective corrosion protection with a low corrosion current density of $5.14 \times 10^{-8} \mathrm{~A} \mathrm{~cm}^{-2}$ and corrosion rate of $1.19 \times 10^{-3} \mathrm{~mm}$ per year, which was attributed to the hydrophobic, inert and dielectric nature of h-BN. However, the low solubility, low functionality and the chemical inertness of h-BN make it difficult to achieve homogenous dispersion in solvents and good compatibility with most polymer matrix. ${ }^{11-15}$ Extensive researches were launched with the aim to improve dispersibility and affinity of the h-BN with polymer matrix. ${ }^{\mathbf{1 1 1 - 1 3 , 1 6 - 1 8}}$ It could be concluded that two primary techniques had been developed for the dispersion of h-BN: covalent and non-covalent methods. ${ }^{4}$ Zhi et al. ${ }^{19}$ reported on the chemical functionalization of BN nanotubes (BNNTs) by introducing hydroxyl groups under a high temperature and high pressure. However, this treatment would generate irreversible structural changes and decrements in electronic and mechanical properties of the BNNTs. In contrast with the chemical functionalization method, noncovalent approach is particularly advantageous because of its less damage to structure, high efficiency and ease of preparation. Gao et al. ${ }^{18}$ presented that h-BN could be well dispersed due to the strong $\pi-\pi$ interaction with peptide. $\mathrm{Wu}$ et $a l^{20}$ reported that hexagonal boron nitride (h-BN) nanoparticles were noncovalently modified with polydopamine in a solvent-free aqueous condition and the as-prepared h-BN/bisphenol E cyanate ester (h-BN/BECy) 
nanos exhibited excellent performance in dynamic-mechanical properties and thermal conductivity.

It is worth noting that the vast majority of researches for h-BN mainly focus on the exfoliation of h-BN with few layers but the concentration of exfoliated $\mathrm{h}-\mathrm{BN}$ with few layers is very low, which means that it is impractical to prepare these thin layers and apply them in the polymer matrix. In addition, there is rarely work on the corrosion protection and tribological performance of the h-BN/polymers. Hence, in this work, the stable dispersion of h-BN nanoplatelets in a variety of organic solvents was achieved by utilizing the productive amine-capped aniline trimer (AT) as dispersant. The possible reason for this stable dispersion is the strong $\pi-\pi$ interaction between the aromatic ring in AT and $\mathrm{h}$ BN. In addition, AT could be chemically incorporated into the epoxy resin through curing reaction of epoxy groups and end amine $\left(-\mathrm{NH}_{2}\right)$, thus resulting in improved h-BN/polymer matrix interfacial interactions. On the other hand, AT has been reported as an electroactive material that can improve the corrosion protection performance of the coating via passivating the metal surface. ${ }^{21-23}$ Hence, we envision the epoxy coating incorporated with h-BN/AT may exhibit better corrosion protection performance than pure epoxy coating owing to the synergistic effect of AT and h-BN. Based on the above advantages, h-BN/epoxy composite coatings with various content of AT dispersed h-BN nanoplatelets were prepared successfully, and their corrosion protection and tribological performance were investigated via electrochemical techniques and a ball-disc tribometer.

\section{Experiments}

\subsection{Materials}

Aniline, $p$-phenylenediamine sulfate, ammonium persulfate, succinic anhydride, petroleum ether, tetrahydrofuran (THF), ammonium hydroxide and concentrated hydrochloric acid $(\mathrm{HCl})$ were purchased from Aladdin Industrial Corporation. Hexagonal boron nitride powders (h-BN, 99\%) were purchased from Sigma-Aldrich. The epoxy resin (E44) and curing agent (650) were purchased from Xuee Resin Factory, Wujiang City and Yuanda Chemical Co. Ltd. China, respectively.

\subsection{Synthesis of aniline trimer (AT)}

The aniline trimer was prepared according to the previous reports. ${ }^{21,22}$ Aniline ( $3.71 \mathrm{~g}$ ) and $p$-phenylenediamine sulfate (5.91 g) were dissolved in $\mathrm{HCl}$ solution $(1.0 \mathrm{M}, 300 \mathrm{~mL})$ with an ice-salt bath at $-5{ }^{\circ} \mathrm{C}$. A solution of ammonium persulfate $(9.08 \mathrm{~g})$ in $\mathrm{HCl}$ solution $(1.0 \mathrm{M}, 250 \mathrm{~mL})$ was dropped into the above-mentioned solution with a dropping funnel. The mixture were stirred for $1 \mathrm{~h}$, then filtered and washed with $\mathrm{HCl}$ solution $(1.0 \mathrm{M}, 200 \mathrm{~mL})$ precooled to $0{ }^{\circ} \mathrm{C}$ to collect the coarse aniline trimer (AT). Then the coarse AT was washed with $\mathrm{NH}_{4} \mathrm{OH}(10 \mathrm{wt} \%)$ and deionized water several times. Finally, the products were dried at $60^{\circ} \mathrm{C}$ in vacuum until constant weight, which were obtained as a blue solid.

\subsection{Fabrication of h-BN/epoxy coatings}

A typical procedure for the preparation of $0.5 \mathrm{wt} \% \mathrm{~h}-\mathrm{BN} / \mathrm{epoxy}$ was described as follows: aniline trimer (AT, $25 \mathrm{mg}$ ) was first dissolved in tetrahydrofuran (THF, $10 \mathrm{~mL}$ ) and sonicated continuously at room temperature for $10 \mathrm{~min}$. Then $50 \mathrm{mg}$ of h-BN powder were added into the above mixtures and sonicated for $30 \mathrm{~min}$. Subsequently, $10 \mathrm{~g}$ of epoxy resin was added to the mixture and sonicated for another $10 \mathrm{~min}$ in order to achieve a homogeneous dispersion. After sonication, THF was removed by rotary evaporation. Finally, $2.5 \mathrm{~g}$ of curing agent was added and stirred for $10 \mathrm{~min}$, whereby degassing was carried out with a vacuum pump at room temperature for $10 \mathrm{~min}$ to remove the air bubbles. The final mixture was coated on the surface of Q235 steel electrodes using a wire bar coater with a thickness of $20 \mu \mathrm{m}$. Coatings were first dried at the room temperature and then at $80{ }^{\circ} \mathrm{C}$ for $2 \mathrm{~h}$. Meanwhile, pure epoxy and h-BN/epoxy coatings containing various content of h-BN $(1.0 \mathrm{wt} \%$ and $2.0 \mathrm{wt} \%$ ) were then prepared in a similar way.

\subsection{Characterization}

To confirm the interaction between the h-BN and AT molecular, UV-Vis absorption spectrum was recorded with a Lambda 950 UV-Vis spectrometer. Meanwhile, Raman spectrum was also measured with a confocal Renishaw invia Reflex Raman spectrometer using the wavelength of $632.8 \mathrm{~nm}$. The morphological features of the h-BN nanoplatelets were estimated by scanning electron microscopy (FESEM, S-4800, HITACHI), scanning probe microscope (SPM, Veeco Dimension 3100V) and transmission electron microscopy (TEM, JEM2100, JEOL, Japan). To obtain the elemental mapping of the h-BN before and after dispersion, the SEM test is conducted by spreading the pristine $\mathrm{h}-\mathrm{BN}$ powder and the $\mathrm{h}$-BN powder dispersed with AT on the conductive adhesive, respectively. The crystal structure of h-BN was identified by XRD (D8 Advance, Bruker AXS, Germany) equipped with $\mathrm{Cu} \mathrm{K} \alpha$ radiation. The $2 \theta$ scan range was from $10^{\circ}$ to $60^{\circ}$ with the scan time of $7 \mathrm{~min}$. The fracture surface of the coatings was examined by SEM (FESEM, S-4800, HITACHI), where the coatings were coated by Au prior by sputtering. The thermal degradation and stability of the coatings were determined by thermo-gravimetric analysis (TG, Pyris Diamond, Perkin-Elmer). An alumina pan was loaded with 5-10 mg of sample material and heated from room temperature to $800^{\circ} \mathrm{C}$ at a rate of $20{ }^{\circ} \mathrm{C} \mathrm{min}^{-1}$ in air flow.

\subsection{Electrochemical measurement}

The electrochemical measurements were conducted in $3.5 \mathrm{wt} \%$ $\mathrm{NaCl}$ solution using an electrochemical work station (CHI660E, Chenhua, Shanghai) to evaluate the corrosion behaviors of the coatings. A conventional three-electrode electrochemical system was used, in which a saturated calomel electrode (SCE), a platinum wire electrode and a specimen (exposed surface area of $1 \mathrm{~cm}^{2}$ ) were used as reference, counter and working electrodes, respectively. The specimen was immersed in $3.5 \mathrm{wt} \%$ $\mathrm{NaCl}$ solution for $30 \mathrm{~min}$ to establish a steady open circuit potential (OCP) prior to the measurements. Long-term electrochemical impedance spectroscopy (EIS) tests were performed at the open circuit potential over a frequency range of $100 \mathrm{kHz}$ to $10 \mathrm{mHz}$, with applied $10 \mathrm{mV}$ sinusoidal perturbations. Zsimpwin software was used for fitting the EIS results. 


\subsection{Tribological test}

Tribological behaviors of the composite coatings were also investigated with a ball-disc tribometer (CSM). Tests were carried out with a sliding frequency of $2 \mathrm{~Hz}$ under constant load of $5 \mathrm{~N}$ over $36 \mathrm{~m}$ distance. The counterpart is made of $316 \mathrm{~L}$ steel ball with diameter of $3 \mathrm{~mm}$. The wear rate and depths of the specimens after tribological test were measured using a surface profiler (D-100, KL $\Lambda$, Tencor). Finally, the surface morphologies of wear tracks were examined by FESEM (S-4800, HITACHI).

\section{Results and discussion}

\subsection{Stable dispersion of $h-B N$ nanoplatelets with the modification of AT and corresponding characterization}

As shown in Fig. 1a, obvious agglomerates and precipitation occurred when h-BN powders were directly dispersed in organic solvents. However, it was worth noting that the h-BN could be stably dispersed in various organic solvents with a concentration of $1 \mathrm{mg} \mathrm{mL}{ }^{-1}$ with the modification of AT (Fig. 1b). The possible reason is that $\mathrm{h}-\mathrm{BN}$ nanoplatelets with a closed hexagonal structure consisting of alternating boron and nitrogen atoms can interact with the aromatic ring of AT through $\pi-\pi$ stacking forces and van der Waals forces among amino moiety. ${ }^{18,20}$

To confirm the speculation of the mentioned above, UV-Vis absorption spectra and Raman spectra were carried out. Fig. 2a showed the Raman spectra of h-BN, AT and AT/h-BN hybrid. Pristine h-BN powder exhibited a sharp characteristic peak at $1365 \mathrm{~cm}^{-1}$, which was ascribed to the $\mathrm{E}_{2 \mathrm{~g}}$ phonon mode. ${ }^{20}$ For AT, the characteristic peaks at 1167, 1406 and $1594 \mathrm{~cm}^{-1}$ were assigned to the $\mathrm{C}-\mathrm{H}$ bending vibrations, phenazine structure and $\mathrm{C}-\mathrm{C}$ stretching of the quinonoid ring, respectively. ${ }^{23}$ After the dispersion of h-BN with AT, h-BN/AT hybrids showed similar peaks with h-BN and AT but the position of the peak varied. It was apparent that the peak corresponding to phenazine structures shifted from $1406 \mathrm{~cm}^{-1}$ (AT) to $1415 \mathrm{~cm}^{-1}$ (AT/h-BN), implying the existence of $\pi-\pi$ interaction between h-BN and AT chains. The UV-Vis absorption spectra of h-BN, AT and AT/h-BN hybrids in THF shown in Fig. $2 \mathrm{~b}$ also provided the direct evidence for the $\pi-\pi$ interaction between AT and h-BN. UV-Vis absorption spectrum of h-BN in THF is featureless. In case of AT, there were three distinct absorption peaks at 285, 332 and $557 \mathrm{~nm}$, which corresponded to the $\pi-\pi^{*}$ transition in benzene unit and the transition of $\pi_{\mathrm{b}}-\pi_{\mathrm{q}}$ from benzene unit to quinone unit, respectively. ${ }^{24}$ Similarly, the AT/h-BN hybrids also exhibited these three absorption peaks at 281, 328 and $557 \mathrm{~nm}$. It was obvious that there was a blue shift about 3-4 $\mathrm{nm}$ for all peaks, which evidenced that the strong charge transfer existed owing to the strong $\pi-\pi$ interactions between the AT and h-BN.

The morphologies of pristine h-BN powder and AT-dispersed h-BN nanoplatelets were compared in Fig. $3 \mathrm{a}$ and $\mathrm{b}$. The pristine h-BN powder was stacked thickly while the AT-dispersed h-BN nanoplatelets with a circular platelet shape and nanometer dimensions were observed after sonication process. According to the previous report, the lattice distance was about $0.35 \mathrm{~nm} .{ }^{1}$ It could be observed from the SPM images in Fig. $3 \mathrm{c}$ that the thickness of AT/h-BN nanoplatelets with thin layers was $\sim 4.0 \mathrm{~nm}$ ( $\sim 11$ layers). Some white particles existed at the surface of h-BN nanoplatelets and Si substrate, which corresponded to the aniline trimer. At the same time, it is inevitable that h-BN thick layers $(20-30 \mathrm{~nm})$ still exist because there is not the use of centrifugation during the whole process (Fig. 3d). In addition, TEM was employed to further analyze the structure of h-BN. Some thin regions could be observed from the lowmagnification TEM and HRTEM images of the h-BN nanoplatelets (Fig. 3e and f). Inset to Fig. 3e showed the electrondiffraction pattern of h-BN nanoplatelets. The pattern revealed a typical six-fold symmetry natural of h-BN. The distances between each two neighboring white dots in Fig. $3 g$ is actually equal to the distances between any two nearest $\mathrm{N}$ or $\mathrm{B}$ atoms. ${ }^{1}$ Lattice fringes with the average distance $0.25 \mathrm{~nm}$ in the inset to Fig. $3 \mathrm{~g}$ were equal to the previous report, indicating that dispersion is a non-destructive modification method. ${ }^{1,11,25}$ From

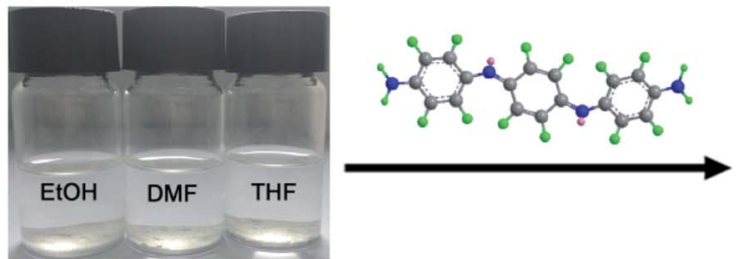

(a)

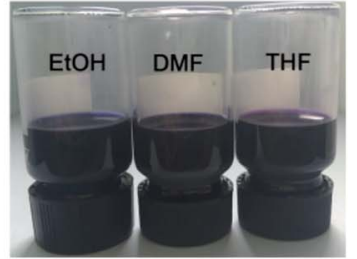

(b)

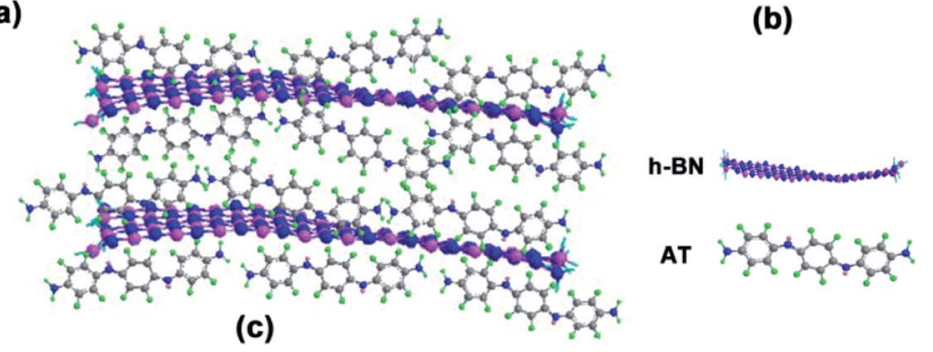

Fig. 1 The dispersion of $\mathrm{h}-\mathrm{BN}$ nanoplatelets in different solvents without (a) or with (b) AT as dispersant and corresponding schematic illustration of $\pi-\pi$ interactions between $\mathrm{h}-\mathrm{BN}$ and $\mathrm{AT}$ (c). 

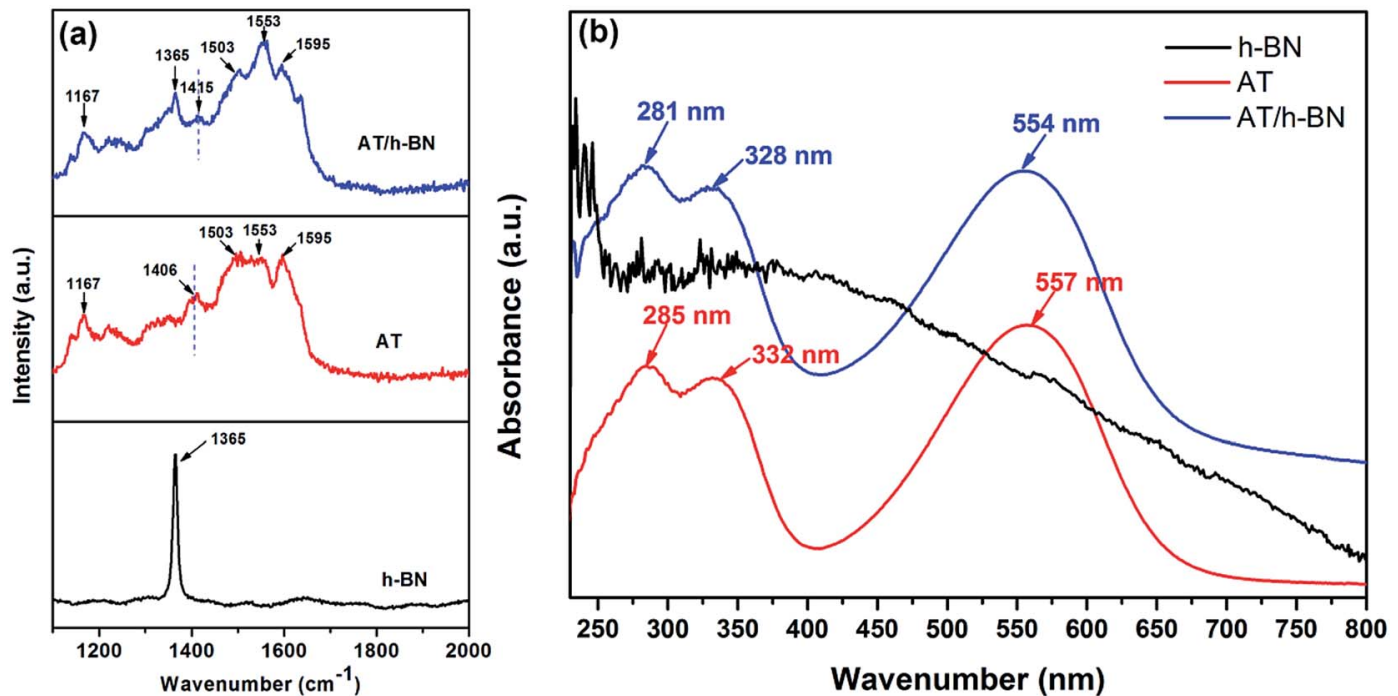

Fig. 2 (a) Raman spectra of $h-B N, A T$ and AT/h-BN hybrids; (b) UV-Vis spectra of $h-B N, A T$ and AT/h-BN hybrids suspension in THF

the above results, it could be concluded that the h-BN powder could be well dispersed in organic solvents with the assistance of AT.

The SEM images and corresponding elemental mapping results of pristine $\mathrm{h}$-BN powder and h-BN powder dispersed with AT are shown in Fig. 4. It can be found from the EDS spectra of selected region 1 and elemental mapping results that the pristine h-BN powder consists of boron (B, $69.13 \mathrm{wt} \%$ ) and nitride ( $\mathrm{N}, 30.87 \mathrm{wt} \%)$. The appearance of the carbon (C) in the mapping results is due to the presence of conductive adhesive at the bottom and margin (Fig. 4a). In case of the h-BN powder dispersed with AT (Fig. 4b), the dispersed h-BN powder with AT are composed of boron (B, $53.05 \mathrm{wt} \%)$, nitride (N, $23.98 \mathrm{wt} \%$ ), carbon (C, $22.23 \mathrm{wt} \%)$ and oxygen $(\mathrm{O}, 0.74 \mathrm{wt} \%)$ owing to the absorption of AT in the h-BN surface (selected region 2). Besides, the distribution of $\mathrm{C}$ is relative homogeneous in the surface of h-BN dispersed with AT but the intensity is weaker than the region with conductive adhesive. Together with the above results, it can be concluded that the existence of AT leads to the well dispersion of h-BN in organic solvents.

Fig. 5 presented the XRD patterns of h-BN, pure epoxy and h-BN/epoxy coating. It could be observed that h-BN exhibited
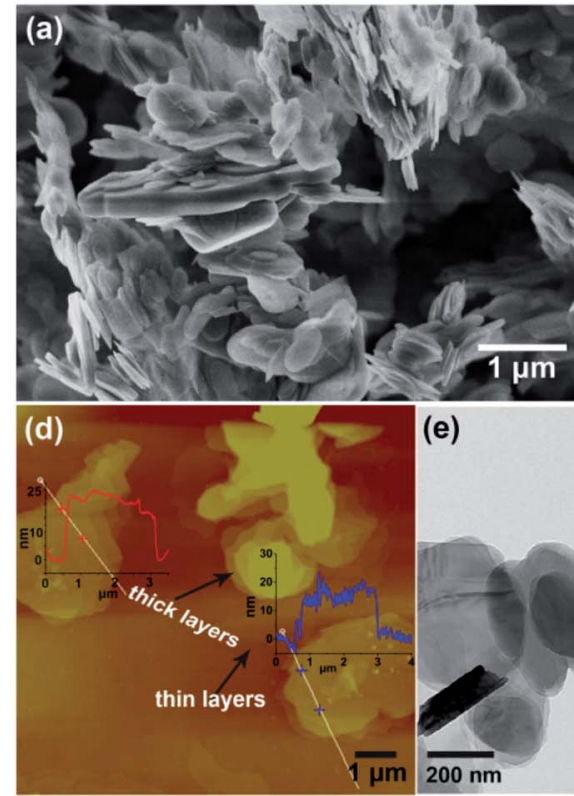

(e)

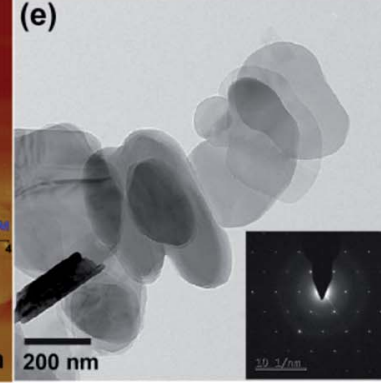

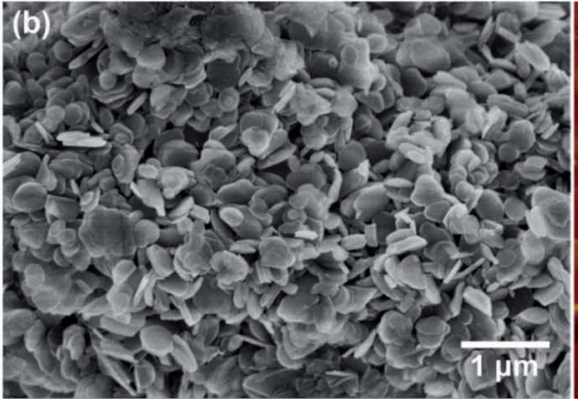

(f)

$50 \mathrm{~nm}$
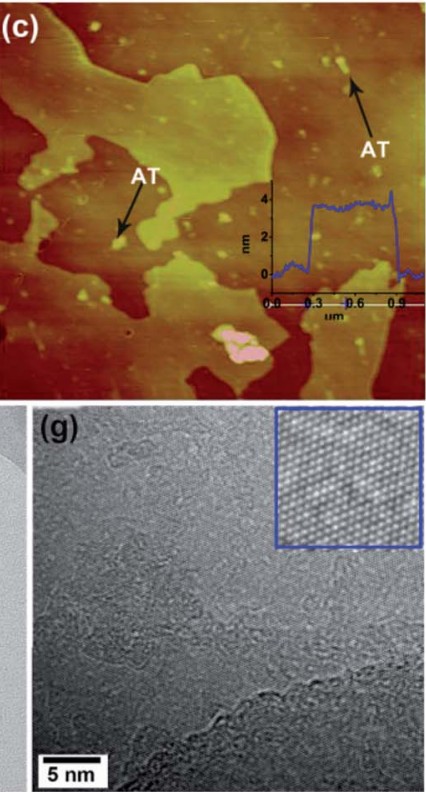

Fig. 3 (a) SEM image of pristine h-BN powder and corresponding EDS spectra, (b) SEM and (c and d) SPM image of h-BN nanoplatelets after the dispersion with AT and corresponding thickness, (e) TEM and ( $f$ and $g$ ) HRTEM images of $h$-BN nanoplatelets with few layers after the dispersion with $A T$, inset to (e) is corresponding electron-diffraction pattern of h-BN nanoplatelets. 

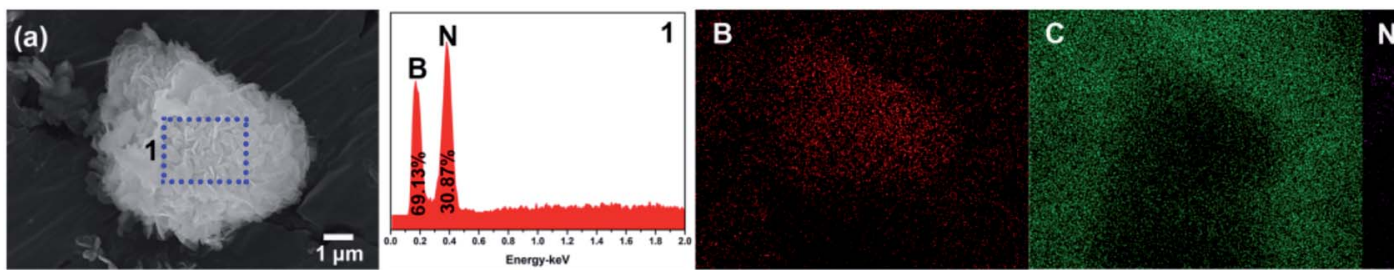

\section{N}
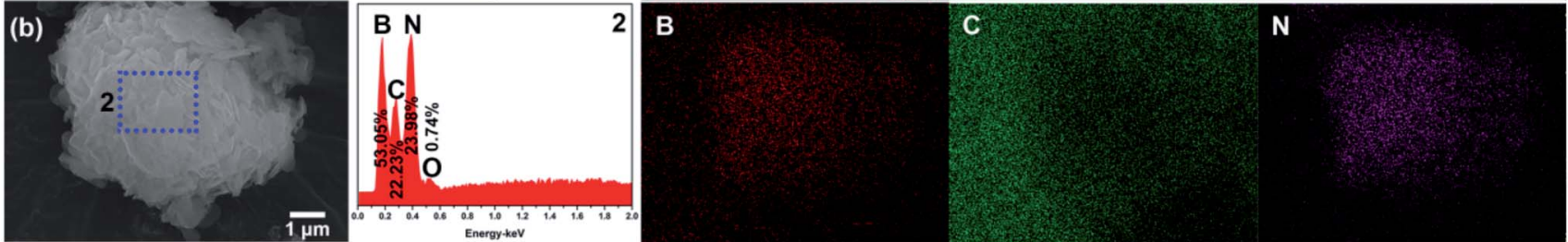

Fig. 4 The SEM images, EDS spectra with selected region and corresponding elemental mapping results of pristine h-BN powder (a) and h-BN powder dispersed with AT (b).

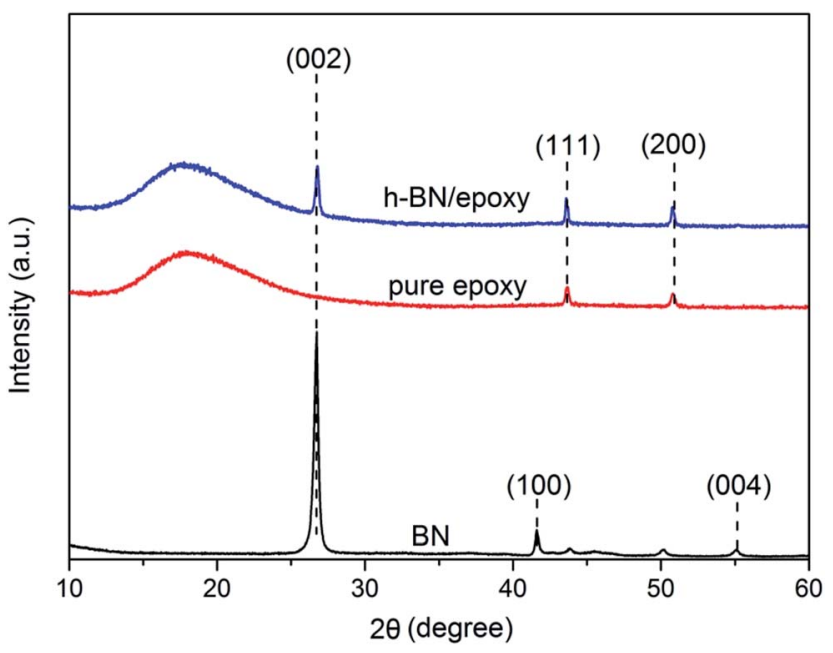

Fig. 5 XRD patterns of h-BN, pure epoxy coating and h-BN/epoxy coating.

a well-crystallized structure with remarkable characteristic peaks corresponding to the (002), (100) and (004) planes. ${ }^{20}$ The (111) and (200) peaks were assigned to the steel substrates. ${ }^{26}$ For pure epoxy coating, a broad peak was observed owing to the amorphous feature of cross-linking in epoxy matrix. ${ }^{27}$ In case of h-BN/epoxy coating, besides the wide peak from epoxy matrices, a weak characteristic peak (002) of h-BN was also observed in the XRD patterns, indicating the intercalation dispersion of $h$ $\mathrm{BN}$ in the epoxy matrix. ${ }^{27,28}$

To investigate the dispersion of h-BN nanoplatelets in the epoxy coating, the fracture surfaces of pure epoxy and h-BN/ epoxy coatings were examined by SEM and TEM, which are shown in Fig. 6. The fracture surface of pure epoxy coating exhibited many oriented bamboo-like fracture patterns initialized from the cracks (Fig. 6a). In contrast, h-BN/epoxy coatings showed quite different fracture morphologies. When $0.5 \mathrm{wt} \%$ of h-BN was incorporated into epoxy resin, the fracture surface of composite coating was smooth with less and shorter cracks as a comparison with pure epoxy coating, which indicated that there was a good dispersion and compatibility between the h-BN nanoplatelets and epoxy matrix. It could be observed from Fig. 6e that h-BN nanoplatelets with few layers were randomly dispersed in epoxy coatings. However, with the increasing content of h-BN nanoplatelets $(1.0 \mathrm{wt} \%$ and $2.0 \mathrm{wt} \%)$, the fracture surface of coatings showed more cracks and defects

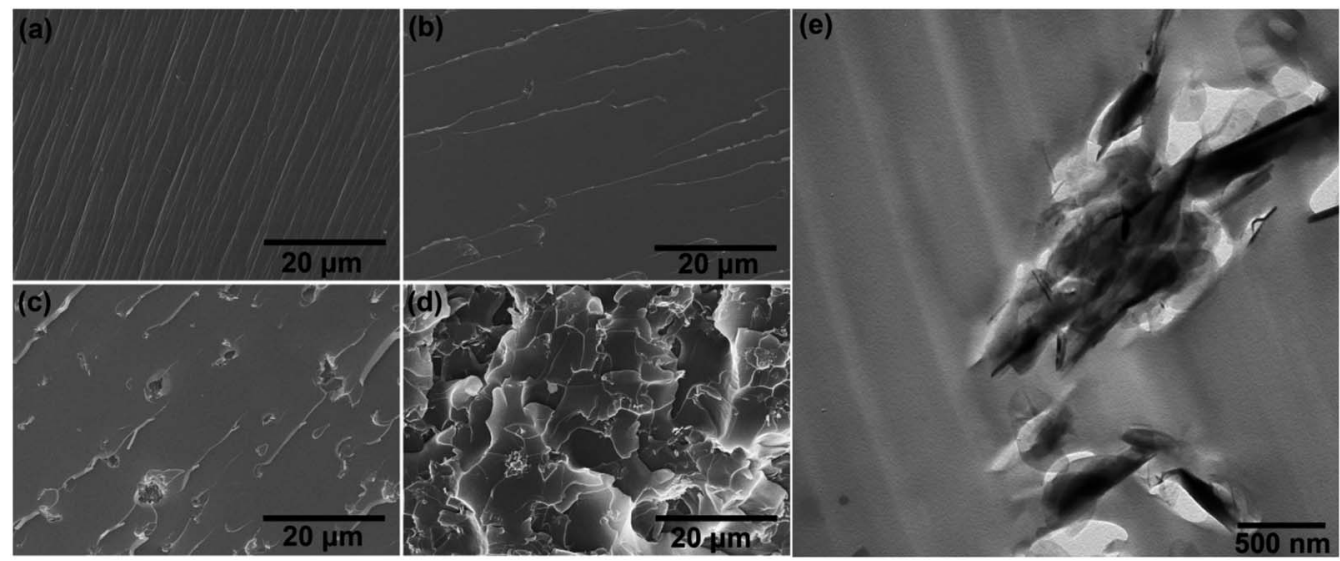

Fig. 6 The SEM images of fracture surface for different samples, (a) pure epoxy, (b) 0.5 wt\% h-BN/epoxy, (c) 1.0 wt\% h-BN/epoxy, (d) 2.0 wt\% hBN/epoxy coatings and (e) TEM image of $0.5 \mathrm{wt} \% \mathrm{~h}-\mathrm{BN} / \mathrm{epoxy}$ coating. 


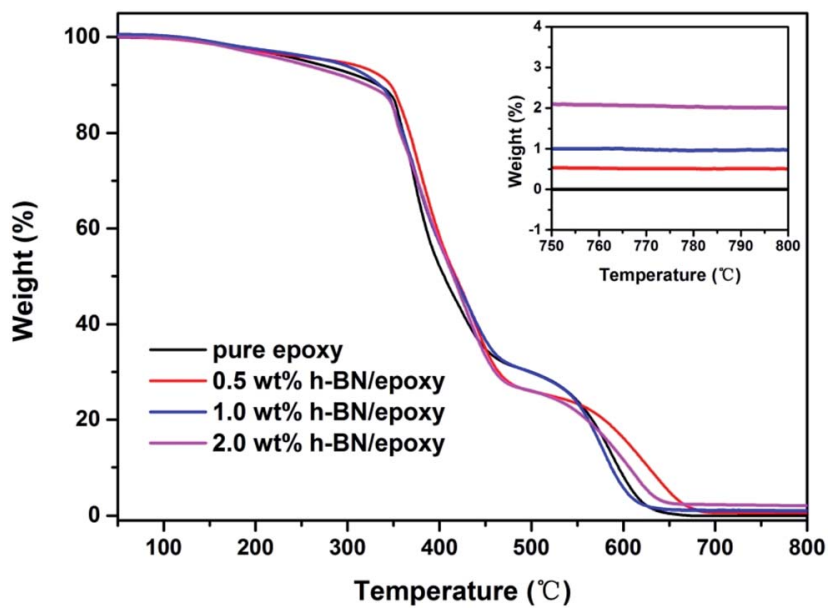

Fig. 7 TGA curves for pure epoxy and h-BN/epoxy coatings as a function of temperature in air atmosphere.

Table 1 Temperature for pure epoxy and h-BN/epoxy coatings at the $10 \mathrm{wt} \%$ and $50 \mathrm{wt} \%$ of weight loss

\begin{tabular}{lll}
\hline Sample & $T_{-10 \%}\left({ }^{\circ} \mathrm{C}\right)$ & $T_{-50 \%}\left({ }^{\circ} \mathrm{C}\right)$ \\
\hline Pure epoxy & 332.8 & 405.1 \\
0.5 wt\% h-BN/epoxy & 346.6 & 417.5 \\
1.0 wt\% h-BN/epoxy & 334.7 & 415.6 \\
2.0 wt\% h-BN/epoxy & 320.6 & 414.7
\end{tabular}

that were probably caused due to the aggregation of h-BN nanoplatelets, as shown in Fig. 6c and d.

TGA was employed to investigate thermal stability and degradation behaviors of the pure epoxy and h-BN/epoxy composites. Fig. 7 displayed the TGA curves of pure epoxy and h-BN/epoxy coatings under nitrogen with a heating rate of $10{ }^{\circ} \mathrm{C} \mathrm{min}^{-1}$ and the detailed data were summarized in Table 1. $T_{-10 \%}$ and $T_{-50 \%}$ are defined as the temperatures where weight loss is $10 \%$ and $50 \%$, respectively. As shown in Fig. 7 , the pure epoxy and h-BN/epoxy composites presented similar degradation behaviors, which consisted of three stages (the vaporization of small molecules, the decomposition of epoxy network and the oxidation of the char residues). ${ }^{29}$ The $T_{-10 \%}, T_{-50 \%}$ and residues at $800{ }^{\circ} \mathrm{C}$ for pure epoxy is $332.8^{\circ} \mathrm{C}, 405.1{ }^{\circ} \mathrm{C}$ and 0 , respectively. Incorporation of $0.5 \mathrm{wt} \% \mathrm{~h}-\mathrm{BN}$ nanoplatelets into epoxy coating results in the increase in $T_{-10 \%}, T_{-50 \%}$ and residues, which is $346.6{ }^{\circ} \mathrm{C}, 417.5{ }^{\circ} \mathrm{C}$ and $0.5 \mathrm{wt} \%$, respectively. However, with the further increase in the content of h-BN nanoplatelets $(1.0 \mathrm{wt} \%$ and $2.0 \mathrm{wt} \% \mathrm{~h}-\mathrm{BN} / \mathrm{epoxy})$, the $T_{-10 \%}$ and $T_{-50 \%}$ decrease gradually with respect to $0.5 \mathrm{wt} \% \mathrm{~h}-\mathrm{BN} /$ epoxy. This implied the thermal stability of the h-BN/epoxy coating was not relevant to the content of h-BN nanoplatelets, but dependent on the dispersion of h-BN in the epoxy matrices. The well-dispersed h-BN nanoplatelets provided an effective barrier to inhibit the mass loss during the thermal degradation process.

\subsection{Anticorrosive performance of the coatings}

Anticorrosive performance of the pure epoxy and epoxy coatings incorporated with different content of h-BN nanoplatelets was investigated in $3.5 \mathrm{wt} \% \mathrm{NaCl}$ solution at various immersion times. The Bode plots of the samples were shown in Fig. 8. At the initial immersion ( 2 days), it could be clearly seen from Fig. 8a that the impedance modulus at lowest frequency $(0.01 \mathrm{~Hz})$ of epoxy coatings incorporated with different content of h-BN nanoplatelets were higher than that of pure epoxy, in which $1.0 \mathrm{wt} \% \mathrm{~h}-\mathrm{BN} / \mathrm{epoxy}$ coating exhibited the highest impedance modulus $\left(\sim 10^{10.5} \mathrm{ohm} \mathrm{cm}^{2}\right)$ among all samples. And the phase angles were almost $90^{\circ}$ at high frequency region and only one time constant was observed in the Bode plots for all samples, which indicated the excellent barrier properties of the coatings at the initial immersion. With the prolonged immersion time, the corrosive medium gradually penetrated into the epoxy matrix. Two time constants could be observed in the Bode plots of the pure epoxy after 20 days immersion, implying that the coating degradation led to the decrease of the barrier properties of the coating, thus allowing the corrosive medium to reach the metal substrate. ${ }^{30}$ Also, impedance modulus of pure epoxy at the lowest frequency $(0.01 \mathrm{~Hz})$ decreased to $\sim 10^{8} \mathrm{ohm}$ $\mathrm{cm}^{2}$ after 60 days immersion. However, in case of the epoxy coating incorporated with different content of h-BN nanoplatelets, there was still only one time constant in the Bode plots during this immersion time and the impedance modulus of $1.0 \mathrm{wt} \% \mathrm{~h}-\mathrm{BN} / \mathrm{epoxy}$ coating had no obvious change. Results indicated that incorporation of h-BN nanoplatelets resulted in the enhancement in the barrier performance of the coating.
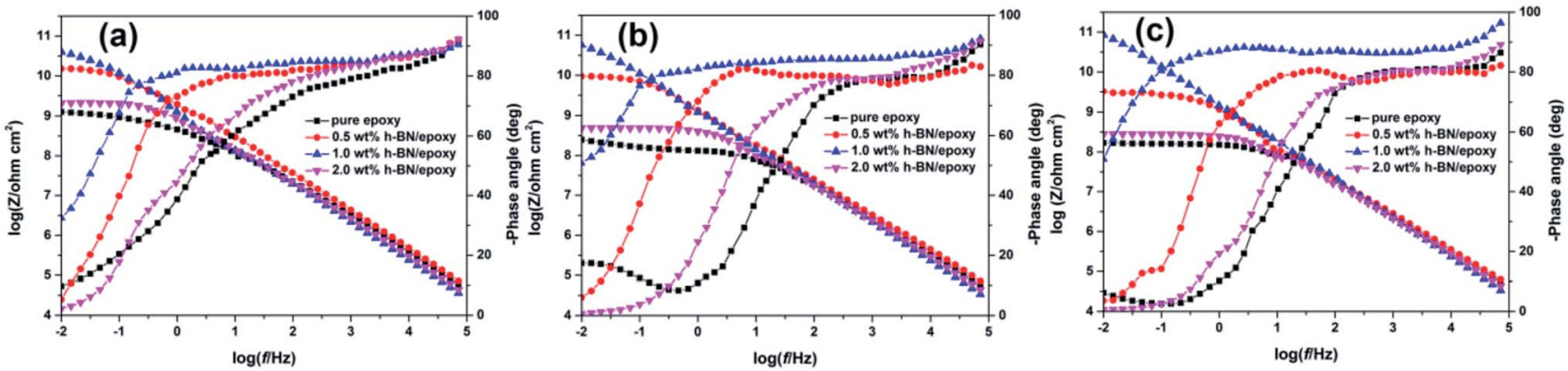

Fig. 8 Bode plots of pure epoxy, 0.5 wt\% h-BN/epoxy, $1.0 \mathrm{wt} \% \mathrm{~h}-\mathrm{BN} / \mathrm{epoxy}$ and $2.0 \mathrm{wt} \% \mathrm{~h}$-BN/epoxy coatings applied on steel substrate after (a) 2 days, (b) 20 days and (c) 60 days exposure to 3.5 wt $\% \mathrm{NaCl}$ solution. 

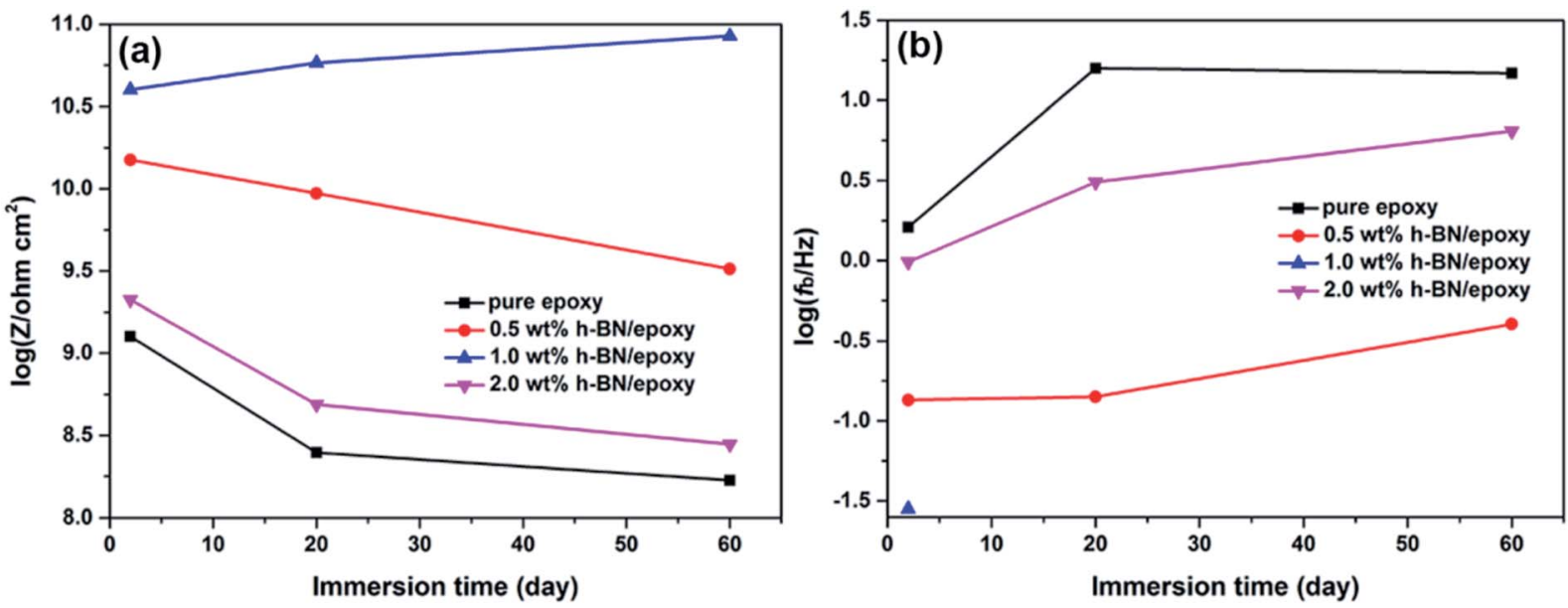

Fig. 9 Variations of (a) $\log Z$ at $0.01 \mathrm{~Hz}$ and (b) $\log f_{\mathrm{b}}$ as a function of immersion time for pure epoxy, $0.5 \mathrm{wt} \% \mathrm{~h}-\mathrm{BN} / \mathrm{epoxy}, 1.0 \mathrm{wt} \% \mathrm{~h}-\mathrm{BN} / \mathrm{epoxy}$ and $2.0 \mathrm{wt} \% \mathrm{~h}-\mathrm{BN} / \mathrm{epoxy}$ coatings in $3.5 \mathrm{wt} \% \mathrm{NaCl}$ solution.

In general, impedance modulus at the lowest frequency $(0.01 \mathrm{~Hz})$ could serve as an estimation of the corrosion protection of the coating. ${ }^{31}$ The variations in impedance modulus at the lowest frequency $(0.01 \mathrm{~Hz})$ of different samples as a function of immersion time were given in Fig. 9a. It could be seen from Fig. 9a that the impedance modulus at the lowest frequency $(0.01 \mathrm{~Hz})$ of all samples decreased with increasing immersion time, indicating the continuous penetration of corrosive medium into the coating matrix. Moreover, it was found that incorporation of h-BN nanoplatelets into the coating resulted in the significant increase of impedance modulus at the lowest frequency $(0.01 \mathrm{~Hz})$ compared to the pure epoxy, especially for $1.0 \mathrm{wt} \% \mathrm{~h}-\mathrm{BN} / \mathrm{epoxy}$ coating. Breakpoint frequency $\left(f_{\mathrm{b}}=45^{\circ}\right)$, was another useful parameter to investigate the effects of h-BN nanoplatelets on the anticorrosive performance of the epoxy coating. The microscopic delaminated areas of the coating were closely related with the variation of $f_{\mathrm{b}}$. Usually, the higher the $f_{\mathrm{b}}$, the larger the delaminated areas of the coating. ${ }^{32}$ The breakpoint frequency $\left(f_{\mathrm{b}}\right)$ of different samples at various immersion times was shown in Fig. 9b. It could be seen from Fig. 9b that the $f_{\mathrm{b}}$ increased and shifted to higher frequencies when the immersion time elapsed. Pure epoxy exhibited the highest $f_{\mathrm{b}}$ and pronounced increase of $f_{\mathrm{b}}$, indicating that the barrier properties of the pure epoxy decreased distinctly with increasing immersion time. In addition, it could be seen that addition of h-BN nanoplatelets to the coating caused lower $f_{\mathrm{b}}$ at all immersion times compared to the pure epoxy. Especially, there was no the phase angle at $45^{\circ}$ from $100 \mathrm{kHz}$ to $0.01 \mathrm{~Hz}$ for the $1.0 \mathrm{wt} \% \mathrm{~h}-\mathrm{BN} / \mathrm{epoxy}$ coating after 20 days immersion, which implied that less coating delamination occurred when h-BN nanoplatelets were incorporated into the epoxy matrix.

Besides, the barrier performance of the coatings could also be evaluated based on the intersection of Bode plots (IBP).$^{33}$
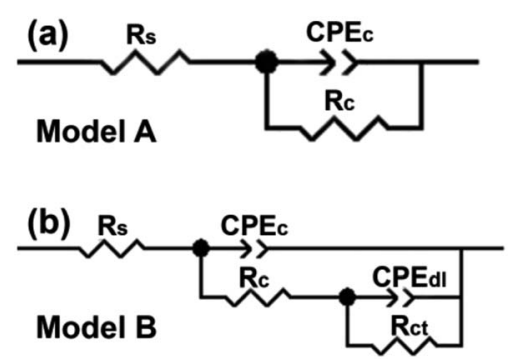

Fig. 11 Equivalent circuits (a) Model $A$ and (b) Model $B$ used to fit the EIS data of pure epoxy and h-BN/epoxy coating.
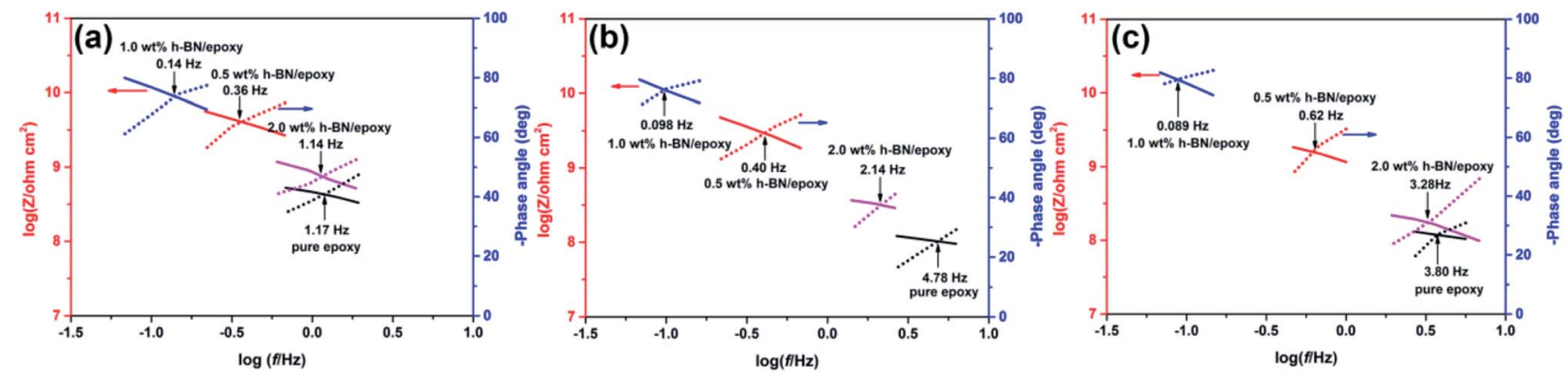

Fig. 10 IBP values of the pure epoxy, 0.5 wt\% h-BN/epoxy, $1.0 \mathrm{wt} \% \mathrm{~h}-\mathrm{BN} / \mathrm{epoxy}$ and $2.0 \mathrm{wt} \% \mathrm{~h}-\mathrm{BN} /$ epoxy coatings immersed in $3.5 \mathrm{wt} \% \mathrm{NaCl}$ solution for (a) 2 days, (b) 20 days and (c) 60 days. 


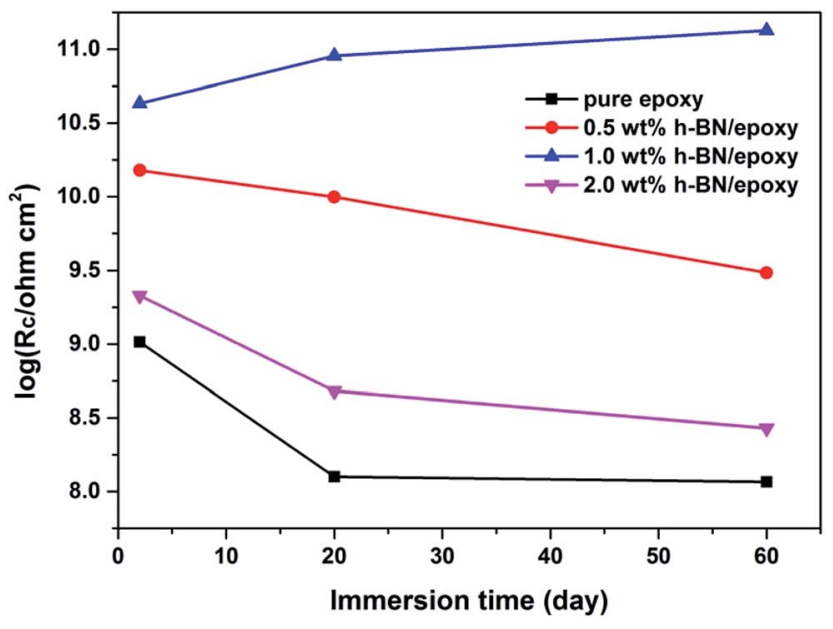

Fig. 12 Time dependence of the coating resistance $\left(R_{\mathrm{C}}\right)$ of different samples immersed in $3.5 \mathrm{wt} \% \mathrm{NaCl}$ solution.

Fig. 10 showed the variation of IBP values with immersion time. It could be seen from Fig. 10 that addition of h-BN nanoplatelets in the epoxy matrix caused the shift of IBP values to lower frequencies compared to the pure epoxy. Moreover, the shift of IBP values to lower frequencies was the most pronounced for $1.0 \mathrm{wt} \% \mathrm{~h}-\mathrm{BN} /$ epoxy coating. In addition, the IBP values shifted to higher frequencies with increasing immersion time for all samples apart from $1.0 \mathrm{wt} \% \mathrm{~h}$-BN/epoxy coating. All of these results indicated that the addition of h-BN nanoplatelets resulted in significant improvement of the barrier properties of the epoxy coating.

To quantitatively assess the anticorrosive property of the h-BN/ epoxy coatings, EIS results were fitted with equivalent circuits. In combination with the Bode plots (Fig. 8), two equivalent electrical circuits (Model A for excellent barrier property of the coating and Model B for active corrosion period of the coating) were put forward to analyze the corrosion process of the coatings as shown in Fig. 11. Where $R_{\mathrm{s}}$ is the solution resistance, and $R_{\mathrm{c}}$ and $R_{\mathrm{ct}}$ correspond to the coating and charge-transfer resistance, respectively. $\mathrm{CPE}_{\mathrm{c}}$ and $\mathrm{CPE}_{\mathrm{dl}}$ represent the coating and doublelayer capacitance, respectively.

For the pure epoxy, the Bode phase plot exhibited one time constant at the initial immersion ( 2 days) and the equivalent circuit at this stage is Model A. Then an additional time constant appeared with the increase of immersion time (20 to 60 days). Obviously, the Model A could no longer fit the data well. Therefore, Model B was employed to interrupt the following process. The time constant at high and middle frequencies is characteristic for the capacitive-resistive response of the coating, and another time constant at the low frequencies reflects the corrosion process taking place at the metal/coating interface. ${ }^{34}$ The occurrence of corrosion process is attributed to the penetration of corrosive medium through defects in the pure epoxy coating. In case of h-BN/epoxy coatings, there was only one time constant (Model A) corresponding
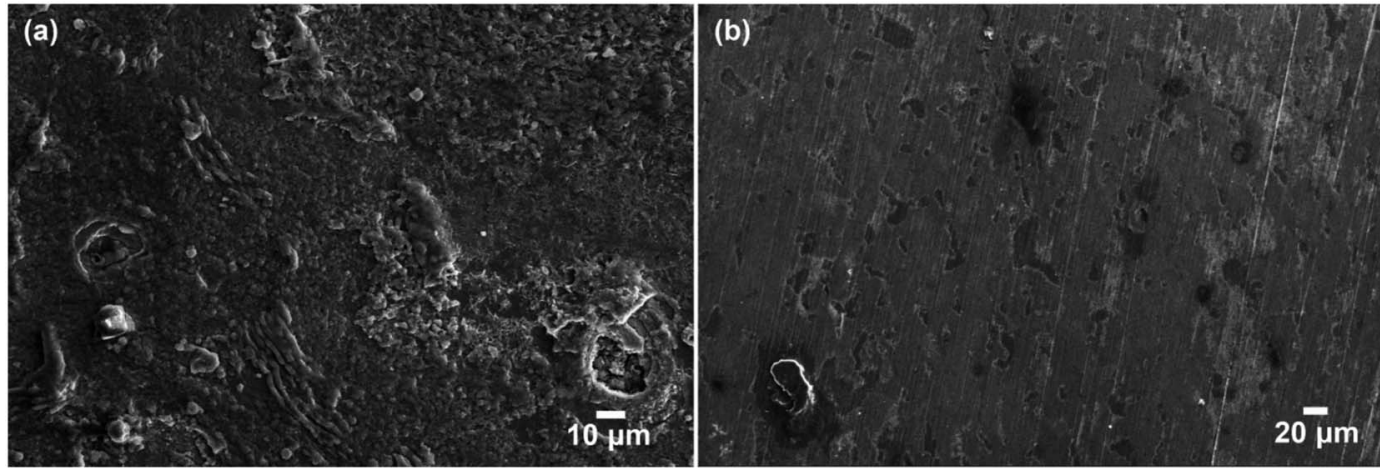

Fig. 13 SEM image of the metal surface after the coating removed at 60 days immersion, (a) metal surface after pure epoxy removed and (b) metal surface after $1.0 \mathrm{wt} \% \mathrm{~h}$-BN/epoxy coating removed.
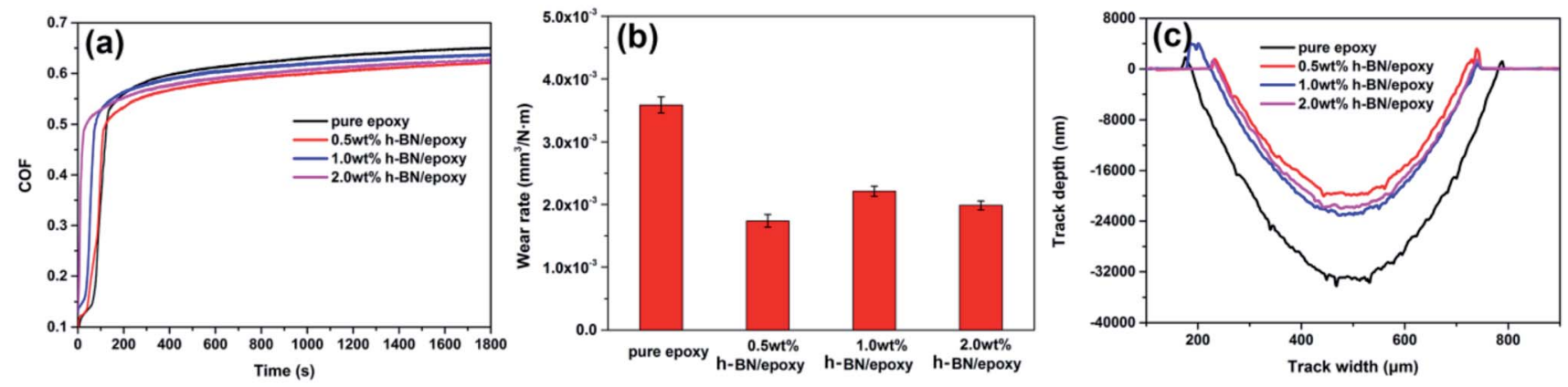

Fig. 14 The variation in friction coefficient (a), wear rate (b) and wear depths (c) for pure epoxy coating and h-BN/epoxy coatings. 
to the capacitive response of the coating during immersion process, which indicated that no corrosion occurred underneath the coatings during 60 days immersion. ${ }^{30}$

Based on the above fitting models, corresponding electrochemical parameters were obtained. Coating resistance $\left(R_{\mathrm{c}}\right)$ is a direct indicator of the corrosion protection performance of the organic coating. ${ }^{35}$ Fig. 12 showed the $R_{\mathrm{c}}$ of the coating as a function of immersion time. The $R_{\mathrm{c}}$ value for pure epoxy decreased sharply with increase of immersion time, which indicated that pure epoxy might rapidly lose its corrosion

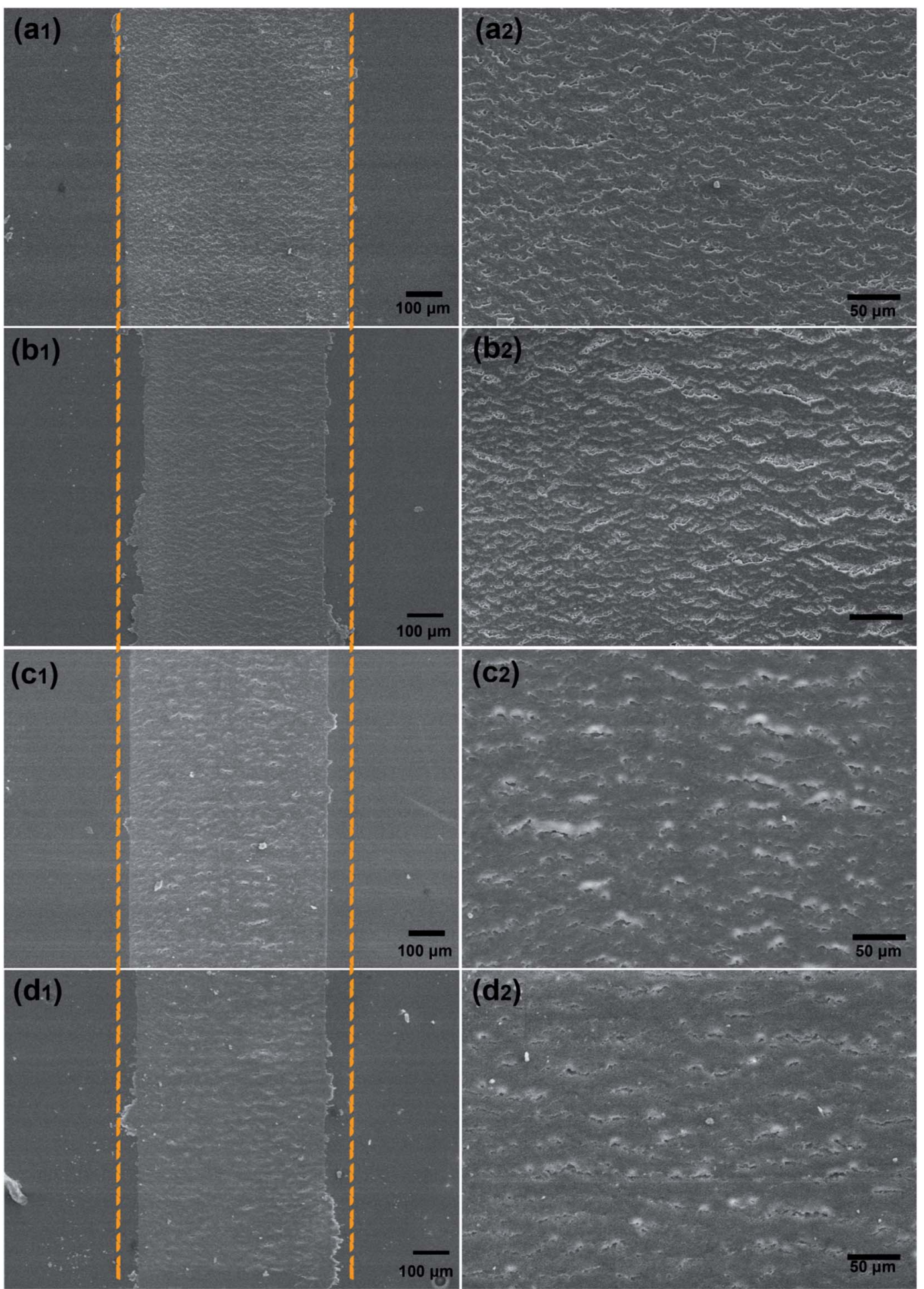

Fig. 15 The surface morphologies of wear tracks for the pure epoxy ( $\mathrm{a} 1$ and a2), and BN/epoxy coatings with different concentration (b1 and b2: $0.5 \mathrm{wt} \%, \mathrm{c} 1$ and c2: $1.0 \mathrm{wt} \%$, d1 and d2: $2.0 \mathrm{wt} \%)$ under dry sliding. 
protection to substrate. Similarly, the $R_{\mathrm{c}}$ value for $0.5 \mathrm{wt} \%$ and $2.0 \mathrm{wt} \% \mathrm{~h}-\mathrm{BN} / \mathrm{epoxy}$ coatings also decreased with the increase in immersion time owing to the continuous penetration of corrosive medium. In particular, the $R_{\mathrm{c}}$ value for $1.0 \mathrm{wt} \% \mathrm{~h}-\mathrm{BN} / \mathrm{epoxy}$ coating varied slightly, which was nearly one thousand orders of magnitude higher than that of pure epoxy after the same immersion time. Hence, it could be concluded from the above results that $1.0 \mathrm{wt} \% \mathrm{~h}-\mathrm{BN} /$ epoxy coating had the best anticorrosive performance among all samples. The existence of dispersed h-BN nanoplatelets could effectively interrupt the crack propagation and reduce the coating porosity, which had a significant influence on the corrosion protection performance of the coating.

From the electrochemical measurements, it can be found that the $1.0 \mathrm{wt} \% \mathrm{~h}-\mathrm{BN} / \mathrm{epoxy}$ coating shows better corrosion protection performance to metal substrate than other coatings. This improvement can be understood from two aspects. First, this improvement is closely related with the dispersion of h-BN in epoxy matrix. If the content of h-BN nanoplatelets is too high, the aggregation of h-BN will occur, thus leading to the generation of micro pores or defects in the coating. In combination with the morphology of fracture surface in Fig. 6, we can find that when the content of h-BN is up to $2.0 \mathrm{wt} \%$, the aggregation of h-BN in epoxy coating becomes severe and this aggregation has a bad effect on the corrosion protection performance of the coating. In case of the epoxy coating incorporated with $0.5 \mathrm{wt} \%$ and $1.0 \mathrm{wt} \% \mathrm{~h}-\mathrm{BN}$, the fracture surface is denser than $2.0 \mathrm{wt} \%$ $\mathrm{h}-\mathrm{BN} / \mathrm{epoxy}$, and hence $0.5 \mathrm{wt} \%$ and $1.0 \mathrm{wt} \% \mathrm{~h}-\mathrm{BN} / \mathrm{epoxy}$ coating has better corrosion protection performance than other coatings owing to their good barrier property from dispersed h-BN. Second, AT used to disperse h-BN can also act as a corrosion inhibitor to passivate the metal surface when corrosive medium reaches to the coating/substrate interfaces. It can be found that the impedance modulus of $1.0 \mathrm{wt} \% \mathrm{~h}$-BN/epoxy coating increases with immersion time, which indicates that AT is already working in inhibiting the corrosion at the coating/ substrate interface. That is to say, the corrosion protection performance of $1.0 \mathrm{wt} \% \mathrm{~h}-\mathrm{BN} / \mathrm{epoxy}$ coating is attributed to the barrier property of the h-BN and the passivated effect from AT.

To visually show the excellent barrier properties, the coatings were peeled off from the metal substrate after 60 days immersion in $3.5 \mathrm{wt} \% \mathrm{NaCl}$ solution. The corrosion status of the metal surface was observed by SEM as shown in Fig. 13. It could be observed from Fig. 13a that the metal surface coated with pure epoxy had been corroded seriously and some corrosion products covered the surface. On the contrary, the metal surface coated with $1.0 \mathrm{wt} \% \mathrm{~h}$-BN/epoxy was almost intact except little region suffered from pit corrosion (Fig. 13b). These results indicated that the dispersed h-BN nanoplatelets with excellent barrier properties delayed the penetration of corrosive medium and prolonged the service life of the metal substrate, which provided a guiding significance to industrial application.

\subsection{Tribological tests}

Fig. 14a showed the variation in friction coefficient for pure epoxy and h-BN/epoxy coatings. As shown in Fig. 14a, the addition of dispersed h-BN nanoplatelets resulted in a slight decrease in friction coefficient and the lowest friction coefficient was found for $0.5 \mathrm{wt} \% \mathrm{~h}-\mathrm{BN} /$ epoxy coating. In addition, the wear rates had also constantly measured using a surface profiler (D-100, KL $\Lambda$, Tencor), and the corresponding results were displayed in Fig. 14b and c. The highest wear rate and depths were found for pure epoxy coating, and the $0.5 \mathrm{wt} \% \mathrm{~h}$ BN/epoxy coating exhibited the lowest wear rate and depths. The reason for the higher friction coefficient and excessive wear was that the increase of temperature caused by the friction led to the soften of pure epoxy coating whereas the stable dispersed h-BN nanoplatelets within epoxy resin (0.5 wt\% h-BN/epoxy coating) resulted in the improvement in thermal stability, which could keep its structural integrity and mechanical properties at higher temperature. ${ }^{36}$ Consequently, the thermal softening of $0.5 \mathrm{wt} \% \mathrm{~h}-\mathrm{BN} / \mathrm{epoxy}$ coating was postponed to higher temperature and $0.5 \mathrm{wt} \% \mathrm{~h}$-BN/epoxy coating exhibited lower friction coefficient and wear rate with respect to pure epoxy coating.

In addition, the surface morphologies of wear tracks for the coatings were further examined by FESEM. It was conspicuous from Fig. 15 that wrinkled wavy morphology and cracks existed on the worn surface for all coatings owing to the thermal effect caused by friction. ${ }^{27,36}$ However, when it came to h-BN/epoxy coatings, less wrinkled wavy and cracks were observed on wear tracks. This was because that the addition of h-BN nanoplatelets improved the thermal stability of epoxy resin and the heat generated during the friction would have slightly effect on the wear of the coating. Thus, h-BN/epoxy coatings showed narrower wear tracks than pure epoxy, which were in agreement with the COF and wear rate results obtained from the tribological test.

From the above discussions, it can be concluded that the h$\mathrm{BN} /$ epoxy coating has good anticorrosive performance and wear resistance due to the barrier properties and thermal stability of h-BN.

\section{Conclusions}

Hexagonal boron nitride (h-BN) nanoplatelets were successfully dispersed in a variety of organic solvents via the functionalization of AT, which was attributed to the strong $\pi-\pi$ interaction between AT and h-BN. Moreover, we have shown the fabrication of h-BN/epoxy coating by curing reaction of epoxy resin, amine harder and AT functionalized h-BN nanoplatelets. Electrochemical and tribological tests were performed to investigate the corrosion protection performance and wear resistance of the epoxy coatings. Conclusions were summarized as follows:

(1) The epoxy coating with $1.0 \mathrm{wt} \%$ of h-BN nanoplatelets provided an effective barrier to the corrosive medium, resulting in superior corrosion protection to the steel substrate. The reason for enhanced corrosion protection of the epoxy coating was the existence of dispersed h-BN nanoplatelets effectively interrupted the crack propagation and reduced the coating porosity, thus inhibiting the penetration of corrosive medium in epoxy resin. AT used to disperse h-BN can also act as a corrosion inhibitor to passivate the metal surface when 
corrosive medium reaches to the coating/substrate interfaces, thus delaying the corrosion of the metal substrate.

(2) The incorporation of h-BN nanoplatelets resulted in the improvement in the wear resistance of epoxy coating and the reduction of friction coefficient under dry conditions. Thermal softening owing to the heat generated during the friction had negative effect on the tribological behavior of polymer coatings, while the addition of h-BN nanoplatelets improved the thermal stability of the epoxy coating, thus leading to lower wear rates and friction coefficient.

\section{Conflicts of interest}

There are no conflicts to declare.

\section{Acknowledgements}

The authors gratefully acknowledged financial support provided by the National Key Basic Research Program (No. 2014CB643302), Strategic Leading Science \& Technology Programme (XDA13040600) and "One Hundred Talented People" of the Chinese Academy of Sciences (Y60707WR04).

\section{References}

1 C. Zhi, Y. Bando, C. Tang, H. Kuwahara and D. Golberg, Adv. Mater., 2009, 21, 2889.

2 H. Shen, J. Guo, H. Wang, N. Zhao and J. Xu, ACS Appl. Mater. Interfaces, 2015, 7, 5701.

3 C.-C. Teng, C. C. M. Ma, K. C. Chiou, T. M. Lee and Y. F. Shih, Mater. Chem. Phys., 2011, 126, 722-728.

4 J. Yu, X. Huang, C. Wu, X. Wu, G. Wang and P. Jiang, Polymer, 2012, 53, 471-480.

5 T. Sainsbury, A. Satti, P. May, Z. Wang, I. McGovern, Y. K. Gun'ko and J. Coleman, J. Am. Chem. Soc., 2012, 134, 18758-18771.

6 M. Yi, Z. Shen, X. Zhao, S. Liang and L. Liu, Appl. Phys. Lett., 2014, 104, 143101.

7 L. Shen, Y. Zhao, Y. Wang, R. Song, Q. Yao, S. Chen and Y. Chai, J. Mater. Chem. A, 2016, 4, 5044-5050.

8 E. Husain, T. N. Narayanan, J. J. Taha-Tijerina, S. Vinod, R. Vajtai and P. M. Ajayan, ACS Appl. Mater. Interfaces, 2013, 5, 4129-4135.

9 Q. Weng, X. Wang, X. Wang, Y. Bando and D. Golberg, Chem. Soc. Rev., 2016, 45, 3989-4012.

10 Z. Liu, Y. Gong, W. Zhou, L. Ma, J. Yu, J. C. Idrobo, J. Jung and A. H. MacDonald, Nat. Commun., 2013, 4, 2541.

11 Y.-T. Liu, X.-M. Xie and X.-Y. Ye, Chem. Commun., 2013, 49, 388-390.

12 R. J. Smith, P. J. King, M. Lotya, C. Wirtz, U. Khan, S. De, A. O'Neill and G. S. Duesberg, Adv. Mater., 2011, 23, 39443948.
13 A. Pakdel, C. Zhi, Y. Bando and D. Golberg, Mater. Today, 2012, 15, 256-265.

14 S. Pal, S. R. C. Vivekchand, A. Govindaraj and C. N. R. Rao, J. Mater. Chem., 2007, 17, 450-452.

15 M. A. Fernandez-Yague, A. Larrañaga, O. Gladkovskaya, A. Stanley, G. Tadayyon, Y. Guo, J. R. Sarasua and S. A. M. Tofail, Bioconjugate Chem., 2015, 26, 2025-2037.

16 S.-Y. Xie, W. Wang, K. S. Fernando, X. Wang, Y. Lin and Y. P. Sun, Chem. Commun., 2005, 29, 3670-3672.

17 C. Zhi, Y. Bando, C. Tang, R. Xie, T. Sekiguchi and D. Golberg, J. Am. Chem. Soc., 2005, 127, 15996-15997.

18 Z. Gao, C. Zhi, Y. Bando, D. Golberg and T. Serizawa, J. Am. Chem. Soc., 2010, 132, 4976-4977.

19 C.-Y. Zhi, Y. Bando, T. Terao, C.-C. Tang, H. Kuwahara and D. Golberg, Chem.-Asian J., 2009, 4, 1536-1540.

$20 \mathrm{H}$. Wu and M. R. Kessler, ACS Appl. Mater. Interfaces, 2015, 7, 5915-5926.

21 C.-J. Weng, C.-H. Chang, C.-W. Peng, S.-W. Chen, J. M. Yeh, C. L. Hsu and Y. Wei, Chem. Mater., 2011, 23, 2075-2083.

22 L. Gu, S. Liu, H.-C. Zhao and H.-B. Yu, ACS Appl. Mater. Interfaces, 2015, 7, 17641-17648.

23 M. Trchová, Z. Morávková, M. Bláha and J. Stejskal, Electrochim. Acta, 2014, 122, 28-38.

24 Y. Guo, A. Mylonakis, Z. Zhang, P. I. Lelkes, K. Levon, S. Li, Q. Feng and Y. Wei, Macromolecules, 2007, 40, 2721-2729.

25 D. Golberg, Y. Bando, L. Bourgeois, K. Kurashima and T. Sato, Appl. Phys. Lett., 2000, 77, 1979.

26 W. Zhu, C.-Y. Nie, C.-H. Ran, Y.-D. Jin and Y. Zhao, Adv. Mater. Res., 2013, 750, 1977-1981.

27 D. Liu, W. Zhao, S. Liu, Q. Cen and Q. Xue, Surf. Coat. Technol., 2016, 286, 354-364.

28 T. T. X. Hang, T. A. Truc, T. H. Nam, V. K. Oanh, J. B. Jorcin and N. Pébère, Surf. Coat. Technol., 2007, 201, 7408-7415.

29 X. Wang, S. Zhou, W. Xing, B. Yu, X. Feng, L. Song and Y. Hu, J. Mater. Chem. A, 2013, 1, 4383.

30 D. Zhang, H. Qian, L. Wang and X. Li, Corros. Sci., 2016, 103, 230-241.

31 T. T. X. Hang, T. A. Truc, N. T. Duong, N. Pébère and M. G. Olivier, Prog. Org. Coat., 2012, 74, 343-348.

32 B. Ramezanzadeh, S. Niroumandrad, A. Ahmadi, M. Mahdavian and M. M. Moghadam, Corros. Sci., 2016, 103, 283-304.

33 B. Ramezanzadeh, E. Ghasemi, M. Mahdavian, E. Changizi and M. M. Moghadam, Carbon, 2015, 93, 555-573.

34 M. Cui, J. Pu, J. Liang, G. Zhang and L. Wang, RSC Adv., 2015, 5, 104829-104840.

35 W. Tian, F. Meng, L. Liu, Y. Li and F. Wang, Prog. Org. Coat., 2015, 82, 101-112.

36 H. Düzcükoğlu, Ş. Ekinci, Ö. S. Şahin, A. Avci, M. Ekrem and M. Ünaldi, Tribol. Trans., 2015, 58, 635-642. 